\title{
Analysis of Radiological Case Presentations and their Impact on Therapy and Treatment Concepts in Internal Medicine
}

\author{
Analyse radiologischer Röntgendemonstrationen und \\ deren Auswirkungen auf Therapie und \\ Behandlungskonzepte in der Inneren Medizin
}

Authors

Lena-Marie Dendl ${ }^{1}$, Andreas Teufel ${ }^{2}$, Stephan Schleder ${ }^{1}$, Janine Rennert ${ }^{1}$, Christian Stroszczynski ${ }^{1}$, Martina Mueller-Schilling ${ }^{2}$, Andreas G. Schreyer

Affiliation

1 Department of Radiology, University Hospital Regensburg, Germany

2 Department of Internal Medicine 1, University Hospital Regensburg, Germany

Key words

decision analysis, cost-effectiveness, health policy and practice

received 4.9.2015

accepted 1.9.2016

Bibliography

DOI http://dx.doi.org/10.1055/s-0042-118884

Published online: 1.3.2017 | Fortschr Röntgenstr 2017; 189: 239-246

(c) Georg Thieme Verlag KG Stuttgart · New York

ISSN 1438-9029

Correspondence

Dr. Lena-Marie Dendl

Department of Radiology, University Hospital Regensburg

Franz-Josef-Strauss-Allee 11

93053 Regensburg

Germany

Tel.: ++49/941/9447418

Fax: $++49 / 941 / 9447409$

lena-marie.dendl@ukr.de

\section{ABSTRACT}

Purpose Evaluation of clinical impact regarding diagnostic and therapeutic changes influenced by interdisciplinary radiological case presentations.

Materials and Methods Prospective evaluation of radiological-gastrointestinal clinical case conferences over a 1 -year period at a tertiary care center. We documented the preparation (phase 1) and clinical case conference (phase 2 ) regarding their impact on the radiology report and further diagnostic work-up and therapy.

Results 1067 examinations were evaluated in 69 clinical case conferences including 487 cases. We calculated a mean time of 35.8 minutes per conference with 5.1 minutes per case for preparation. During phase 1 , major changes compared to the previous report were found in $1.2 \%$ of cases, and no change was found in $91.4 \%$ of cases. In phase
2 an additional relevant finding was found in $0.6 \%$ of cases, while there was no major change to the reports in $99 \%$ of cases. We recommended further radiological diagnostic workup in $9 \%$ of cases and interventional radiological examination in $2.7 \%$ of cases, while no change was documented in $83.2 \%$. Further radiological or surgical therapy was recommended in $7 \%$ and $6.8 \%$ of cases, respectively. There was no change in therapy in $78.5 \%$ of cases.

Conclusion The analysis of an interdisciplinary radiological case presentation in internal medicine shows that the case discussion with the radiologist results in a change in patient management in $37.3 \%$ of cases ( $16.8 \%$ diagnosis, $21.5 \%$ therapy). Overall, interdisciplinary radiological clinical case conferences help to improve the management and quality of patient care. Our data support the broad implementation of radiological clinical case conferences.

\section{Key Points}

- The second opinion obtained during the preparation of a radiological case presentation does not change the written report in most cases.

- "Talking radiology" in radiological case presentations results in a significant change in patient management in over $1 / 3$ of all cases.

- In radiological clinical case conferences an experienced radiologist can initiate diagnostic and interventional radiological methods that can be correctly implemented in therapeutic pathways.

- "Talking radiology" improves the quality of therapy and patient care.

\section{Citation Format}

- Dendl L. M., Teufel A., Schleder S. et al. Analysis of Radiological Case Presentations and their Impact on Therapy and Treatment Concepts in Internal Medicine. Fortschr Röntgenstr 2017; 189: 239-246

\section{ZUSAMMENFASSUNG}

Ziel Evaluation des zeitlichen Aufwandes sowie der diagnostischen und therapeutischen Entscheidungen durch eine interdisziplinäre Röntgendemonstration.

Material und Methode Prospektive Auswertung von radiologisch-gastrointestinalen Röntgenbesprechungen eines Universitätsklinikums über 1 Jahr. Wir dokumentierten, inwieweit Vorbereitung (Phase 1) und Besprechung (Phase 2) den radiologischen Befund und weitere Diagnose und Therapie beeinflussen.

Ergebnisse Bei 69 internistischen Röntgenbesprechungen mit 487 Fällen wurden 1067 Untersuchungen demonstriert. Die durchschnittliche Vorbereitungszeit lag bei 35,8 Minuten mit 5,1 Minuten pro Fall. Während der Phase 1 wurde in 1,2\% der Fälle ein zusätzlicher relevanter, bisher nicht diagnostizierter Befund entdeckt, während sich in 91,4\% keine Befundänderung ergab. In Phase 2 wurde zu 0,6\% ein 
weiterer relevanter Befund entdeckt, in $99 \%$ der Fälle wurde keine Änderung dokumentiert. Nach der gemeinsamen klinischen Diskussion wurde in $9 \%$ der Fälle weitere radiologische Bildgebung und bei 2,7\% weitere interventionell radiologische Diagnostik indiziert, während bei $83,2 \%$ keine Änderungen vorgenommen wurde. Bezüglich therapeutischer Veränderungen wurde bei $7 \%$ die Indikation zur weiteren radiologischen Therapie und bei $6,8 \%$ zur chirurgischen Therapie gestellt, bei 78,5\% erfolgte kein Wechsel des Therapieregimes.

Schlussfolgerungen Die Analyse einer radiologischen interdisziplinären Röntgendemonstration in der Inneren Medizin zeigt, dass durch die Falldiskussion mit dem Radiologen in 37,3\% eine Änderung des Behandlungskonzeptes (16,8\% Diagnose, 21,5\% Therapie) veranlasst wird. Zusammenfassend zeigen unsere Daten eine Verbesserung der Patientenversorgung und Qualität der Behandlung durch interdisziplinäre radiologische Konferenzen und unterstützen deren breite Implementierung.

\section{Introduction}

Radiological case presentations or rounds are clinical case conferences in which radiological examinations from the clinical routine are presented by a radiologist to the relevant clinical partners. Following the presentation of radiological findings, the cases are additionally discussed by the treating physicians in attendance and further diagnostic or therapeutic decisions are made. As a result of the interaction between radiologists and managing physicians during these radiology rounds or case presentations, a decision is able to be made in a short discussion in the clinical routine in an efficient and focused manner. Additional diagnostic or therapeutic measures are often also determined in these discussions. In contrast to tumor boards, radiological case presentations in clinical case conferences include only two clinical partners, namely the presenting radiologist and the treating managing physician. Tumor boards, on the other hand, are multidisciplinary and are often limited, due to the specialization of the participants, to individual organ regions or specific clinical issues [1].

Radiology rounds are a firmly established routine at hospitals and take place daily in some large departments in a ritualized manner. Although radiological case presentations and discussions of findings are an important and fixed part of clinical radiology in terms of "talking radiology", there is currently a lack of more precise data regarding the time required for preparation and presentation. From a radiological standpoint, it must be determined whether the often time-intensive preparation results in sufficient changes in diagnostic and therapeutic pathways and thus supports better patient care. These clinical case conferences also provide radiologists with the opportunity to obtain additional important information about patients and thus to increase report quality and work satisfaction in radiology as a result of better clinical involvement in diagnostic and therapeutic pathways. In addition, it is important for radiologists to be able to indicate or influence important diagnostic as well as therapeutic and interventional decisions directly in the clinical case conferences. A third advantage is potential improvement of result quality since the initial radiology report is reevaluated by an additional experienced and specialized colleague during preparation and can be improved in a further step in the joint clinical case conference by consolidating all relevant clinical information and comments from the treating physicians.

From the numerous radiological case conferences at our hospital, we selected a daily discussion covering a broad range of nonsurgical care for further evaluation. The goal of this study is to use a systematic prospective analysis of radiological case presentations in a clinical case conference in the areas of gastroenterology, endocrinology, rheumatology, hepatology, intensive care medicine, and infectology at a maximum care hospital (university hospital) to evaluate the extent to which the preparation of radiology rounds by a radiologist specialist as additional reporting improves radiology reports. A second part of the analysis is intended to evaluate the extent to which the radiological discussion in the presence of the primary managing physicians with consolidation of all relevant information results in a subsequent change in terms of a subsequent supplementary report and the extent to which the radiological case presentation affects the further diagnostic and therapeutic approach. In addition to aspects of possible improvement of the quality of results and structure, the time required for radiology rounds and the preparation of radiological case presentations is to be systematically documented and evaluated.

\section{Materials and methods}

The documentation and evaluation of the radiological case conference and its effect on diagnosis and treatment were approved by the responsible ethics commission (reference number 15-1600287). Prospective data acquisition was performed over a period of 12 months between August 2014 and August 2015. For this purpose, we evaluated daily clinical rounds with radiological case presentations in internal medicine with a focus on hepatology, gastroenterology, infectology, endocrinology, and rheumatology at a maximum care hospital (university hospital). The radiological case presentation is performed every workday between 14:00 and 14:45. Managing physicians in internal medicine must register interesting cases to be discussed with the radiological images in writing with specification of the patient name, disease, and issue at least 3 hours prior to the scheduled radiological case conference. Registration is performed via fax. In addition, it is possible to present and discuss external images, i. e., images acquired at a radiology department outside of the hospital, in the radiological case presentation. The original report of the externally acquired images, which are typically cross-sectional images such as CT or MRI, must be included with the request. The preparation and implementation of radiological case presentations is performed by the head physician of the radiology department or a radiology specialist. Only radiological case presentations held by the same radiologist with abdominal imaging experience were prospectively included in our evaluation. The discussion after the radiological case presentation regarding the effect on diagnosis and treatment was also held by a single internist with a specialization in gastroenterology and hepatology so that consistency with respect to radiology and internal medicine can be assumed in the data analysis.

The radiological case presentation was prepared after creation of a radiological case presentation list in the PACS (Picture Archiv- 
- Table 1 Evaluation of radiological examination - number of changes (with percentage) with respect to the written preliminary report on the basis of the preparation of the radiological case presentation (phase 1) and the presentation and discussion of the cases (phase 2 ) in 487 cases and 1067 radiological examinations over 1 year.

\begin{tabular}{|c|c|c|c|c|c|}
\hline & $\begin{array}{l}\text { Rx } \\
\text { no written preli- } \\
\text { minary report } \\
\text { available }\end{array}$ & $\begin{array}{l}\text { Ro } \\
\text { no change to the writ- } \\
\text { ten preliminary report }\end{array}$ & $\begin{array}{l}\text { R1a } \\
\text { additional finding } \\
\text { (not clinically rele- } \\
\text { vant) }\end{array}$ & $\begin{array}{l}\text { R1b } \\
\text { additional finding } \\
\text { (clinically rele- } \\
\text { vant) }\end{array}$ & $\begin{array}{l}\text { R2 } \\
\text { "downgrading" } \\
\text { with respect to } \\
\text { the written } \\
\text { preliminary } \\
\text { report }\end{array}$ \\
\hline phase 1 & $35(7.2 \%)$ & 445 (91.4\%) & $0(0 \%)$ & $6(1.2 \%)$ & $1(0.2 \%)$ \\
\hline phase 2 & $\mathrm{n} / \mathrm{a}$ & 482 (99\%) & $1(0.2 \%)$ & $3(0.6 \%)$ & $1(0.2 \%)$ \\
\hline
\end{tabular}

ing and Communication System) typically approximately 2 hours prior to the radiological case presentation. All radiological imaging methods for the particular patients desired for the presentation were prepared by the radiologist for this purpose. The internal or external radiology reports were viewed and the case and the further diagnostic and therapeutic approach were reevaluated.

This preparation phase of the radiological case presentation was referred to as phase 1 in the analysis. The date of the radiological case conference, the names and birth dates of the patients as well as the primary disease(s) documented on the requests were documented. Retrospective sorting of the most frequently occurring issues and diseases with subsequent coding and classification as tumors (A), vascular diseases (B), chronic inflammation and diseases (C), acute inflammation or abscesses (D), and radiological evaluation with an unclear focus or bleeding with an unclear origin (E) was performed after completion of the study inclusion in August 2015. The number of CT, MRI, and angiographic examinations and the time needed by the radiologist to view the examinations and prepare for each patient in phase 1 was prospectively documented. The time needed for preparation of radiological examinations not performed and interpreted at the own hospital was documented separately. For the evaluation of internal and external radiology reports, written documentation was performed. In the case of a lack of a written report of current examinations from the own hospital, a note was made in the Rx column ( $\vee$ Table 1). In the case of an own evaluation identical with the existing written preliminary report, the case was documented as R0. Clinically non-relevant secondary findings were classified as R1a and additional relevant main findings as R1b. If the available written report was considered to be too sensitive with respect to content the diagnoses were "downgraded", this was documented in column R2.

In the case of presentation of externally acquired images, the quality of both the images and the reports was evaluated according to subjective criteria of the presenting physician. The images were considered diagnostically sufficient in the case of AR-Qa and as insufficient to initiate additional diagnostic and therapeutic consequences in the case of AR-Qb, typically indicating the need for a repeat examination. External reports were documented as ARO in the case of agreement of the external report with the own opinion analogously to the system of radiological reporting mentioned above. Otherwise, AR1 or AR2 was documented.
Phase 1 was evaluated using descriptive statistics. Therefore, the average time needed for preparation as well as the number of presented cross-sectional imaging methods (CT, MRI, angiography) were recorded cumulatively.

Phase 2 was defined to evaluate the influence of radiological case presentations during clinical rounds. The radiological images were presented by the radiologist in the clinical rounds in phase 2 . Following presentation of the particular patient from the viewpoint of the managing physicians, a discussion was conducted among all present assistant physicians, specialists, senior physicians and senior culsultant regarding the further diagnostic and therapeutic approach. Changes to the diagnostic and therapeutic approach were documented in writing by the responsible managing physicians for chart rounds. At the end of these 45-minute radiology rounds, the presenting radiologist and the experienced internist performed a joint, written evaluation of the extent to which the radiology report or the radiological case presentation changed the further diagnostic and therapeutic approach.

To document the influence of this complementary clinical information on the radiology report, the information from $>$ Table 1 was analyzed again with respect to a change to the initial written report (R0, $R 1 a, R 1 b, R 2$, see above).

To evaluate the clinical effects of the radiological case presentation, the diagnostic consequences for the individual patients were documented. Therefore, there was no relevant change in the further diagnostic course of action in the case of D0 ( $\vee$ Table 2). The completion of further radiological examination (D1a) and further interventional radiological examination such as tissue puncture or angiographic intervention (D1b) was additionally noted. Further options were the implementation of additional technical examination (e.g. echocardiography, pulmonary function, etc.) (D2), the implementation of additional endoscopic examination (D3), and the implementation of supplementary lab tests (D4), as well as other diagnostic investigation not described further (D5).

With respect to therapeutic consequences, no relevant change in treatment based on the clinical case conference was documented as T0 ( $\triangleright$ Table 3$)$. Additional radiological therapy, for example angiographic or CT-guided interventions such as local ablation or drainage therapy, was designated as T1. Additional endoscopic therapies (T2), additional surgical therapies in terms of an operation (T3), and changes to medication (T4) were also noted. A decrease in therapy following the radiological case conference was 
- Table 2 Evaluation of diagnostic consequences - change to the further diagnostic approach in 487 cases over one year on the basis of radiological case presentations in percentage.

\begin{tabular}{|l|l|l|l|l|l|}
\hline $\begin{array}{l}\text { D0 } \\
\text { no change to } \\
\text { further } \\
\text { diagnostic } \\
\text { approach }\end{array}$ & $\begin{array}{l}\text { D1a } \\
\text { indication } \\
\text { for further } \\
\text { radiological } \\
\text { imaging }\end{array}$ & $\begin{array}{l}\text { D1b } \\
\text { indication for } \\
\text { further inter- } \\
\text { ventional } \\
\text { radiological } \\
\text { examination } \\
\text { (puncture, an- } \\
\text { giography, etc.) }\end{array}$ & $\begin{array}{l}\text { D2 } \\
\text { indication } \\
\text { for further } \\
\text { technical } \\
\text { examination }\end{array}$ & $\begin{array}{l}\text { D3 } \\
\text { indication } \\
\text { for further } \\
\text { endoscopic } \\
\text { examination }\end{array}$ & $\begin{array}{l}\text { D4 } \\
\text { indication } \\
\text { for further } \\
\text { lab testing }\end{array}$ \\
\hline $\begin{array}{l}\text { giother diagnos- } \\
\text { tic investigation }\end{array}$ \\
\hline $83.2 \%$ & $2.7 \%$ & $1.6 \%$ & $2.9 \%$ & $0.4 \%$ & $0.2 \%$ \\
\hline
\end{tabular}

- Table 3 Evaluation of therapeutic consequences - change with respect to therapeutic consequences in a total of 487 cases on the basis of the discussion in the radiological case presentation in percentage.

\begin{tabular}{|l|l|l|l|l|l|}
\hline $\begin{array}{l}\text { T0 } \\
\text { no major change in } \\
\text { treatment on the basis } \\
\text { of the radiological case } \\
\text { conference }\end{array}$ & $\begin{array}{l}\text { T1 } \\
\text { indication for } \\
\text { further radiologi- }\end{array}$ & $\begin{array}{l}\text { T2 } \\
\text { indication for } \\
\text { endoscopic } \\
\text { treatment }\end{array}$ & $\begin{array}{l}\text { T3 } \\
\text { indication } \\
\text { for surgical } \\
\text { treatment }\end{array}$ & $\begin{array}{l}\text { T4 } \\
\text { indication for } \\
\text { treatment with } \\
\text { medication }\end{array}$ & $\begin{array}{l}\text { T5 } \\
\text { indication for a } \\
\text { treatment re- } \\
\text { duction }\end{array}$ \\
\hline $78.5 \%$ & $7.0 \%$ & $2.4 \%$ & $6.8 \%$ & $3.7 \%$ & $1.6 \%$ \\
\hline
\end{tabular}

documented as T5. Documentation of a change in diagnostic approach and treatment was performed in consensus between the radiologist leading the clinical case conference and the responsible internist following the conference.

In the case of only descriptive statistics, data collection and evaluation were performed using a table calculation (Microsoft Excel for Mac 2016, Version 15.13.1, Microsoft, WA, USA).

\section{Results}

\section{Patient group}

The patient group presented in the radiological case presentation included 487 individual cases with an average patient age of 59 years (range: 18 to 91 years). The majority of the presented patients had a tumor disease $(48 \%, \mathrm{n}=235)$ followed by acute inflammatory changes $(20 \%, n=98)$. Chronic diseases were present in $18 \%$ of cases $(n=90)$. In $13 \%$ of cases $(n=61)$ the reason for presentation in the joint case conference was an unclear inflammatory or bleeding focus.

\section{Number of cases, examinations, and preparation times}

In the prospective one-year evaluation between August 2014 and August 2015, 69 radiology rounds were included in the clinical case conferences in internal medicine with a focus on hepatology, gastroenterology, infectology, endocrinology, and rheumatology and were conducted by the same radiologist and internist. In total, 487 cases were discussed and evaluated in the 69 clinical case conferences. The 487 presented cases included a total of 1067 ra- diological examinations. $69.5 \%(n=742)$ of these examinations were CT examinations, $26.4 \%(n=282)$ were MRI examinations, and $4.0 \%(n=43)$ were angiographic examinations or interventions. The average daily preparation time for the conference was 35.8 minutes (range: $9-83$ minutes). On average, 7.1 patients (range: 0 - 15) were discussed in each case conference. An average of 10.8 CT scans (range: 1 - 40), 4.1 MRI scans (range: 0 11 ) and 0.6 angiographic examinations (range: $0-7$ ) were presented each day.

The average preparation time was 5.1 minutes (range: $1-20$ minutes) for each presented case. On average, 1.5 CT examinations (range: $0-7$ ), $0.6 \mathrm{MRI}$ examinations (range: $0-4$ ), and 0.1 angiographic examinations (range: $0-4$ ) needed to be processed for each case.

The average preparation time for externally acquired radiological images was 4.6 minutes (range: 1 - 20 minutes) per conference day.

\section{Analysis of the preparation phase (phase 1)}

No written or verbal preliminary report was available during preparation of the radiological case presentation (phase 1) in 35 of the 487 evaluated cases ( $\triangleright$ Table 1 ). In 6 cases (1.2\%), an additional relevant and presentation-worthy finding (R1b) that was not mentioned in the written preliminary report was discovered by the presenting radiologist. This included three cases of patients who had Crohn's disease as the primary disease and consequently underwent MR enterography examination. A non-specific intestinal wall thickening had been described in the original written report but was subsequently evaluated by the presenting radi- 
ologist as a specific inflammatory correlate based on diffusion imaging. Bony changes showing an $8 \mathrm{~mm}$ osteolysis of the thoracic spine in one case and an osteoporotic collapsed vertebral body of the lumbar spine in a second case had not been included in the written report for the $\mathrm{CT}$ scans of two patients but were detected during preparation of the radiological case conference. In a further case, enlarged hilar lymph nodes in known sarcoidosis were not mentioned in the written report but were identified during preparation.

Downgrading, i. e., interpretation of a finding as having a lower clinical impact than in the written report (R2), occurred in only one case $(0.2 \%)$ during preparation of the radiological case presentation. In this case, necrotizing pancreatitis had been diagnosed in a 91-year-old patient in the written report but was interpreted by the presenting radiologist as exudative pancreatitis without necrosis.

In 445 of 487 cases (91.4\%), no change in the radiology report (R0) was made by the presenting radiologist compared to the written preliminary report.

Externally acquired scans were evaluated in a total of 64 cases in 69 case conferences. 60 of these scans were considered qualitatively sufficient. In 4 cases, the images were considered qualitatively insufficient or inadequate with respect to the performance of the radiological examination so that the presenting radiologist recommended repeating the examination. An MRI examination with additional contrast agent phases and diffusion-weighted sequences had to be performed in four cases of liver imaging to diagnose suspicious liver masses. There was no relevant discrepancy regarding the content of the reports for the 64 externally performed examinations. Downgrading of the external report on the basis of our own internal reporting occurred in only one case. In this case, a finding that had been interpreted externally as a malignant liver tumor was diagnosed as an uncomplicated FNH in the radiological case conference.

\section{Analysis of the radiological case conference phase (phase 2)}

In total, the radiological case presentation resulted in a change of the radiological diagnosis compared to the written preliminary report in 5 of 487 presented patients ( $\triangleright$ Table 1 ). An additional, non-relevant secondary finding (R1a) was discovered in one case as a result of the conference and additional clinical information. In this case older, consolidated rib fractures were discovered on the basis of the presented patient history but were neither clinically apparent nor of therapeutic relevance. In a total of three cases, additional clinically relevant findings ( $\mathrm{R} 1 \mathrm{~b}$ ) were identified during the joint discussion. In one case the in-depth case discussion identified a bone metastasis on the non-contrast head CT scan that had not been mentioned during primary reporting. In another case an L2 compression fracture was identified in the case conference upon patient complaint of pronounced back pain. The third case was a thoracic soft tissue hematoma in an intensive care patient that had been overlooked during primary reporting and was detected during the radiological case presentation under consideration of clinical information regarding a quickly progressing local mass bordering a recently introduced thoracic drain ( $\bullet$ Fig. 1). The case conference resulted in downgrading of the report in only one case. The diagnosis of a regenerative nodule coud be made in one patient with liver cirrhosis and hepatocellular carcinoma by comparing previous scans brought to the conference under consideration of the constant finding.

Regarding the further diagnostic approach, there was no relevant change with respect to the decision for new diagnostic methods in 405 of 487 cases (83.2\%) ( $>$ Table 2 ). Additional diagnostic radiological methods were indicated in $9 \%$ of cases $(n=44)$, and additional interventional diagnostic radiological methods such as CT-guided histological examination or interventional angiographic examinations were recommended in $2.7 \%$ of cases $(n=13)$. Additional technical diagnostic methods were decided upon in $1.6 \%$ of cases $(n=8)$ and additional endoscopic examination were initiated in $2.9 \%$ of cases $(n=14)$. Additional lab tests were indicated in two patients and "other diagnostic investigation" (D5, - Table 3), i. e., nuclear medicine imaging via PET-CT, was indicated in one patient. In total, additional diagnostic modalities were therefore indicated by the radiological case conference in $16.8 \%$ of the presented patients.

With respect to further therapeutic consequences, there was no acute change to the therapeutic approach in $78.5 \%$ of cases ( $n=382$ ) based on the radiological case presentation. In $7.0 \%$ of cases $(n=34)$, a further radiological treatment, e. g. local ablation (RFA, etc.) or an angiographic intervention (e. g. TACE, TIPSS, etc.) was recommended during the radiological case presentation. An additional endoscopic therapy was recommended in $2.4 \%$ of cases $(n=12)$ and additional surgical treatment in the form of surgery was recommended in $6.8 \%$ of cases $(n=33)$. In $3.7 \%$ of cases $(n=18)$, the interdisciplinary case conference resulted in a change in treatment with additional medication and in $1.6 \%$ of cases $(n=8)$ the radiological case presentation resulted in a reduction of the extent of the further therapeutic approach or the therapeutic measures were reduced.

With regard to externally acquired images presented in the radiological case presentations, a joint decision was made in a total of five cases to repeat the externally acquired scans due to insufficient image quality or a lack of important sequences or contrast agent phases.

\section{Discussion}

Radiological case presentations during clinical rounds at a university hospital for internal medicine with a focus on hepatology, gastroenterology, infectology, endocrinology, and rheumatology were prospectively evaluated and analyzed in the present study. 69 radiological case presentations with 487 individual cases and 1067 radiological examinations presented by the same radiologist were analyzed over a period of one year. CT examinations (69.5\%) were performed more frequently than MRI examinations (26.4\%). The average daily preparation time of approximately 36 minutes calculated as image preparation time, not including time spent on organizational activities and any necessary post-processing, together with the time required for the actual radiological case presentation results in a time commitment of an experienced radiologist of over 1 hour per day for a radiological case presentation performed in the clinical routine in gastroenterology and hepatology. It must be noted here that approximately 5 - 10 radio- 

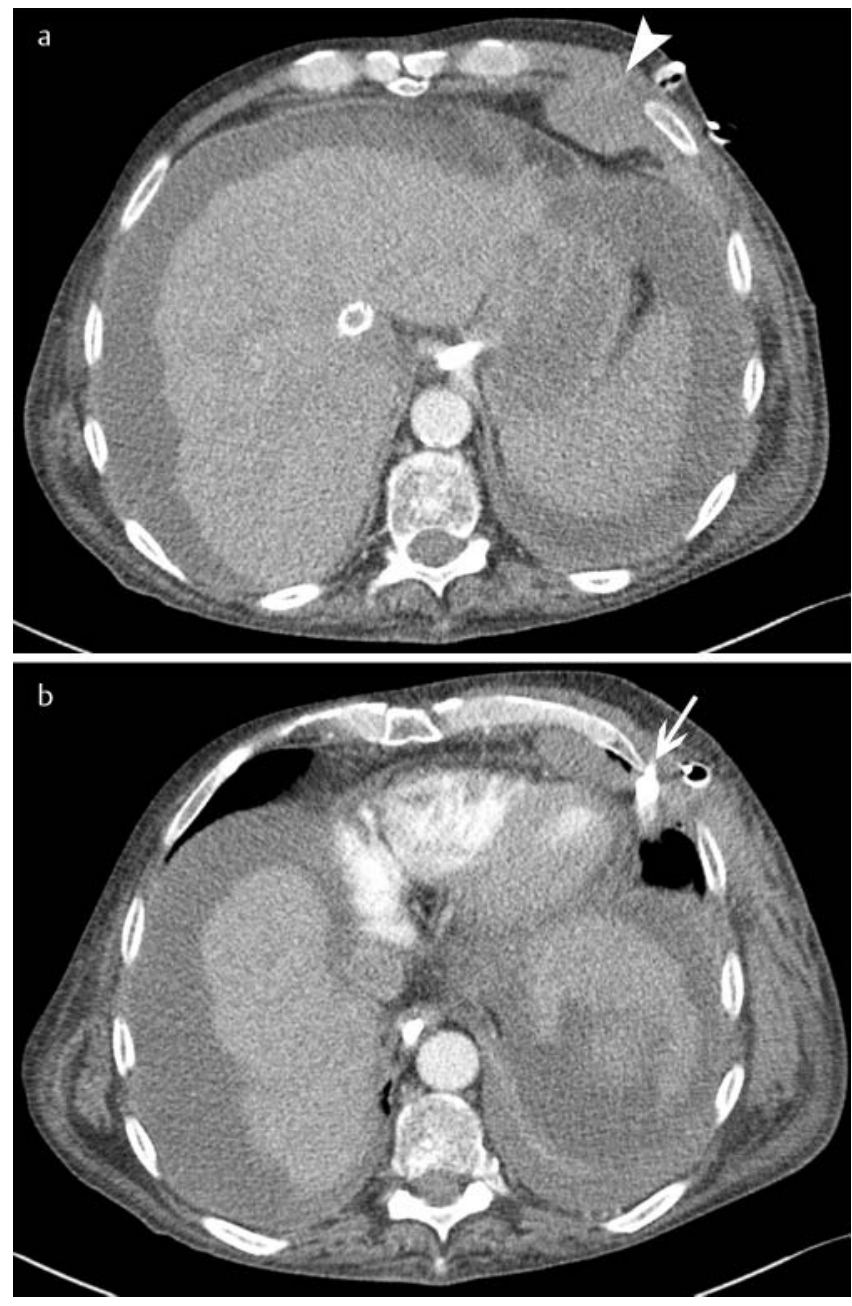

- Fig. 1 A hematoma of the left anterior thoracic wall (arrow tip) was first seen on an axial contrast-enhanced CT scan of an intensive care patient $\mathbf{a}$. In the discussion following the radiological case presentation, the managing physician in internal medicine noted that after placement of a thoracic drain (arrow) new thoracic swelling and bleeding was noticed at this location $\mathbf{b}$ so that the important finding of acute hematoma was able to be added based on the clinical discussion.

logical case presentations are offered daily during clinical rounds in the clinical routine at a maximum care hospital in addition to the numerous tumor boards. Therefore, an analysis by McDonald et al. in 2013 determined the following breakdown of the main workload of a radiologist doing clinical work: reporting (35\%), radiological interventions (23\%), training young colleagues (15\%), conducting clinical case conferences (14\%), informal case presentation (10\%) [2].

The hypothesis that the review and preparation of radiological examinations by a specialist prior to radiological case presentations typically after a written report is already available improves the quality of reports could not be proven by our results. There is no significant change to preliminary findings in $91.4 \%$ of cases and additional clinically relevant findings are discovered in $1.2 \%$ of cases in the preparation phase. In $7.2 \%$ of examinations for which a written report is not yet available at the time of prepara- tion for the radiological case presentation, the preparing physician must perform independent reporting thus increasing the time expenditure.

In relation to the very low portion of relevant report changes, it must be assumed as a limitation that complete new reporting is not performed by radiology specialists in the clinical reality during preparation of radiological case presentations. In most cases the initial report is used. Independent new reporting can only be performed on a report/problem-oriented basis due to the high number of examinations in a radiological case presentation. The number of radiological findings changed as a result of the subsequently performed joint discussion of the radiological images shows that no relevant change to the written preliminary report is made in up to $99 \%$ of cases. Important additional findings are revealed by supplementary clinical information given by the colleagues in charge of patient care in less than $1 \%$ of cases. The results therefore show a very satisfactory agreement of initial radiology reports with the final assessment following the interdisciplinary clinical case presentation. However, it must be noted at this point that consultation with the primary managing physicians often takes place during initial report generation so that, for example, possible differential diagnoses can be better evaluated and put in context particularly in complex intensive care cases.

In addition, as noted above, it must be assumed in our examined patient group that additional reporting during the preparation of radiological case presentations is not performed as an expert second opinion but rather that the preparation of a radiological case presentation is largely based on trust in one's own institution and the high quality of the preliminary reports in one's own department. Radiological case presentations and changes to radiology reports and results based on the communicative or discussion-based part of a radiological case presentation and the preparation thereof have not yet been systematically analyzed in the literature to our knowledge. There are only studies analyzing the influence of a highly specialized radiology second opinion on the further management of tumors or special diseases. In a study in 2013, Lysack et al. examined the difference between external primary radiology reporting and reporting of a neuroradiologist regarding tumor staging in 94 patients with verified head and neck tumors [3]. In this study the tumor staging was changed based on the expert opinion of the neuroradiologist in $56 \%$ of cases and the recommended tumor management was changed in $38 \%$ of cases. In a study by Eakens in 2012, a highly specialized subgroup of pediatric radiologists was examined regarding the effect of the their second opinion [4]. A lack of agreement between the initial report and the second opinion of the pediatric radiologists was detected here in $41.8 \%$ of cases in 773 examined radiology reports. According to the authors' own definition, $21.7 \%$ of these cases showed a decisive lack of agreement. The authors conclude that there can be a significant discrepancy with respect to second opinions in pediatric radiology between $12.6 \%$ in neuroradiological imaging and $32.6 \%$ in imaging of the rest of the body. However, the examined gastroenterology and hepatology patients represent a main focus of the hospital so that the initial reports are already on a high level and there are no comparable discrepancies as in the above-mentioned studies in the comparison between external reports of secondary and ter- 
tiary centers and the reporting of highly specialized radiologists in neuroradiology and pediatric radiology.

To our knowledge, there is also no systematic evaluation in the literature with respect to an analysis of the influence of radiological case presentations in clinical rounds on the further diagnosis of patients. In our study, there was no relevant change in the further diagnostic approach in $83.2 \%$ of patients so that the diagnostic cascade for these patients appeared to be concluded after the case conference in the radiological case presentation. The indication for further radiological examinations was determined in $11.7 \%$ of all patients, resulting in a direct recommendation by the radiologist for a further purely diagnostic, noninvasive, radiological, usually complementary method in $9.0 \%$ of the presented patients. The new indication or recommendation by the radiologist resulted in further interventional radiological examination in $2.7 \%$ of patients. In total, the further diagnostic approach was able to be concretely defined in $16.8 \%$ of all presented patients on the basis of the radiological case conference and image presentation.

A limitation of our own study is that it could not be definitively analyzed whether the implementation of further diagnostic investigation was the exclusive result of the discussion in the radiological case conference. It would hypothetically also be possible that decisions based solely on the written radiology report would have resulted in an identical further approach in some cases. On the whole, it can be assumed based on the known high clinical influence of radiological case conferences on the further diagnostic and therapeutic cascade that the end points achieved in our study (change in treatment concept, additionally ordered examination) are representative. This is the case in particular in difficult cases at a tertiary care center [1].

Regarding a change in the therapeutic approach, similar numbers as in further diagnostic investigation were present. In $78.5 \%$ of patients, no relevant change was made to the therapeutic approach on the basis of the radiological case conference. Further radiological treatment was indicated in $7.0 \%$ of the discussed patients. This included, for example, local ablative methods in liver masses, drainage of abscesses, or angiographic treatment approaches such as placement of a TIPSS. An important reason for radiological case conferences is certainly clinical since interventional-radiological methods can also be offered by radiologists with interventional competency and discussed together with the colleagues providing clinical care. There was also an indication for further surgical therapeutic methods in $6.8 \%$ of the examined cases. It was thus able to be shown that timely and efficient changes in therapeutic management were able to be achieved based on the radiological examination together with the discussion of the cases with the colleagues providing clinical care. To our knowledge, the literature does not contain an analysis of radiological case conferences regarding therapeutic methods and a change in patient management. Only analyses of tumor boards performed on a multidisciplinary basis with participation of radiologists are available. Thus, in their analysis, Chafe et al. describe the influence of pathologists in gynecological tumor boards as decidedly important [5]. The pathological report was changed in $33 \%$ of cases solely on the basis of the preparation for the tumor board in this publication in which 414 patients were retrospective- ly analyzed and a change of patient management was achieved in the tumor board in $12 \%$ of cases. In a prospective study regarding the clinical influence of multidisciplinary tumor boards of neck tumors, 120 patients were prospectively examined. In this study it was concluded that the tumor board resulted in a change of the tumor diagnosis, staging, and treatment plan in 27 patients [6]. Similar to the tumor board results cited here, it could also be shown in our study that the joint patient case conferences resulted in a change of patient management in terms of a diagnostic or therapeutic change in $37.3 \%$ of cases. As in the case of tumor boards, it can be assumed that an improvement of patient care can be achieved by the joint clinical discussion between managing physicians in internal medicine and experienced radiologists.

\section{Summary}

In our study an attempt was made for the first time to systematically analyze radiological case presentations in clinical rounds with a focus on gastroenterology and hepatology with respect to radiology as well as to determine the effects of radiological case presentations on further diagnostic investigation and treatment. We were able to determine that the additional analysis of radiological images by the presenting physician both in the preparation phase and in the radiological case conference with the managing clinical colleagues typically results in an additional relevant diagnosis in only a minimal number of cases, indicating good quality of the primary radiology report. However, a change in patient management was able to be achieved on the basis of the joint radiological case conference in $38.3 \%$ of cases (16.8\% diagnostic investigation, $21.5 \%$ treatment). Particularly with respect to the therapeutic consequences seen in $21.5 \%$ of cases, it is notable regarding clinical relevance that the radiological case conference resulted in a change to surgical interventions in $6.8 \%$ of cases and in a therapeutic change to radiological interventions with a therapeutic approach in up to $7.0 \%$ of cases.

In summary, it can be concluded that interdisciplinary radiological-clinical case conferences result in a change of patient management in over $1 / 3$ of discussed cases and represent an improvement in patient care and treatment quality in the clinical routine.

Clinical relevance of the study

- A second opinion in the form of case preparation by an experienced radiologist changed the primary radiological report in only approximately $1 \%$ of cases.

- As a result of interdisciplinary radiological case conferences in gastrointestinal internal medicine, a change in treatment regime was able to be achieved in over $1 / 3$ of cases.

- Interdisciplinary radiological case conferences definitely help to improve patient care by integrating knowledge and all relevant patient information.

Conflict of interest

The authors declare that they have no conflict of interest.. 


\section{Literatur}

[1] Lamb BW, Green JS, Benn J et al. Improving decision making in multidisciplinary tumor boards: prospective longitudinal evaluation of a multicomponent intervention for 1421 patients. J Am Coll Surg 2013; 217: $412-420$

[2] MacDonald SL, Cowan IA, Floyd RA et al. Measuring and managing radiologist workload: a method for quantifying radiologist activities and calculating the full-time equivalents required to operate a service. J Med Imaging Radiat Oncol 2013; 57: 551-557
[3] Lysack JT, Hoy M, Hudon ME et al. Impact of neuroradiologist second opinion on staging and management of head and neck cancer. J Otolaryngol Head Neck Surg 2013; 42: 39

[4] Eakins C, Ellis WD, Pruthi S et al. Second opinion interpretations by specialty radiologists at a pediatric hospital: rate of disagreement and clinical implications. Am J Roentgenol 2012; 199: 916 - 920

[5] Chafe S, Honore L, Pearcey R et al. An analysis of the impact of pathology review in gynecologic cancer. Int J Radiat Oncol Biol Phys 2000; 48: $1433-1438$

[6] Wheless SA, McKinney KA, Zanation AM. A prospective study of the clinical impact of a multidisciplinary head and neck tumor board. Otolaryngol Head Neck Surg 2010; 143: 650-654 